\title{
A proof of Pesin's formula
}

\author{
RICARDO MAÑÉ† \\ Instituto de Matemática Pura e Aplicada, Rio de Janeiro, Brazil
}

(Received 2 October 1980)

Abstract. Let $f: M \rightarrow M$ be a diffeomorphism of a compact manifold $M$ and let $\chi: M \rightarrow R$ be defined by putting $\chi(x)$ equal to the sum of the non-negative characteristic exponents of $f$ at $x$, each being counted with its multiplicity. If $\mu$ is an $f$-invariant probability of $M$ which is absolutely continuous relative to Lebesgue measure, then Pesin has proved the entropy, $h_{\mu}(f)$, is given by $h_{\mu}(f)=\int_{M} \chi d \mu$. We prove this formula without using the theory of stable manifolds.

The Lyapunov exponents of a diffeomorphism $f$ of a compact boundaryless manifold $M$ are defined by Oseledec's theorem which states that, for any $f$-invariant probability measure $\mu$ on $M$, for almost every point $x \in M$ there exists a unique family of numbers $\lambda_{1}(x)>\cdots>\lambda_{m}(x)$ (the Lyapunov exponents of $f$ at $x$ ) and a unique splitting $T_{x} M=E_{1}(x) \oplus \cdots \oplus E_{m}(x)$ such that

$$
\lim _{n \rightarrow \pm \infty}(1 / n) \log \left\|\left(D_{x} f^{n}\right) v\right\|=\lambda_{j}(x)
$$

for all $0 \neq v \in E_{i}(x), 1 \leq j \leq m$. The entropy $h_{\mu}(f)$ and the Lyapunov exponents are connected by the inequality [2]

$$
h_{\mu}(f) \leq \int_{M} \chi d \mu
$$

where $\chi$ is defined by

$$
\chi(x)=\sum_{\lambda_{i}(x) \geq 0} \lambda_{j}(x) \operatorname{dim} E_{i}(x) .
$$

Pesin's formula [1] states that, when $\mu$ is absolutely continuous with respect to the Lebesgue measure of $M$ and the derivative of $f$ is Hölder continuous, then (1) is an equality

$$
h_{\mu}(f)=\int_{M} \chi d \mu
$$

The proof of this result given by Pesin relies heavily on the stable manifold theory, especially in the absolute continuity of the measurable foliation given by the stable

† Address for correspondence: Dr R. Mañé, Instituto de Matemática Pura e Aplicada, Rua Luiz de Camões 68, 20060 Rio de Janeiro, RJ, Brazil. 
manifolds. The purpose of this note is to give a different, simpler proof of this result which does not make use of the stable manifolds. Clearly the stable manifold theory is of great intrinsic interest and its absolute continuity properties are a fundamental tool in the remarkable theory that Pesin developed for the case when (besides the hypothesis of (2)) the Lyapunov exponents are $\neq 0$ a.e. Nevertheless, it seems interesting to have a proof of (2) exhibiting the simple, elementary nature of this formula.

Our proof will be based on a general lower estimate for metric entropy that makes it possible to avoid the use of partitions. Let us suppose that $g: M \supset$ is a map and $\rho: M \rightarrow(0,1)$ a function. If $x \in M$ and $n \geq 0$, define

$$
S_{n}(g, \rho, x)=\left\{y \mid d\left(g^{j}(x), g^{j}(y)\right) \leq \rho\left(g^{i}(x)\right), 0 \leq j \leq n\right\} .
$$

If $\mu$ is a measure on $M$ and $g$ and $\rho$ are measurable, define

$$
h_{\mu}(g, \rho, x)=\limsup _{n \rightarrow+\infty}(1 / n)\left(-\log \mu\left(S_{n}(g, \rho, x)\right)\right) .
$$

Proposition. If $g: M 2$ is measurable, $\mu$ is a g-invariant probability measure on $M$ and $\nu \gg \mu$ is another measure on $M$ (not necessarily g-invariant), then

$$
h_{\mu}(g) \geq \int_{M} h_{\nu}(g, \rho, x) d \mu(x)
$$

for every function $\rho: M \rightarrow(0,1)$ such that $\log \rho$ is $\mu$-integrable.

The proof of this proposition requires several lemmas.

LEMMA 1. If $\sum_{1}^{\infty} x_{n}$ is a series with $0<x_{n}<1$ for all $n$ such that $\sum_{1}^{\infty} n x_{n}<+\infty$, then

$$
\sum_{1}^{\infty} x_{n} \log \left(1 / x_{n}\right)<+\infty \text {. }
$$

Proof. Let $S$ be the set of integers $n \geq 1$ such that $\log \left(1 / x_{n}\right)<n$. Then $n \notin S$ implies $x_{n} \leq \exp (-n)$. Hence

$$
\sum_{1}^{\infty} x_{n} \log \left(1 / x_{n}\right) \leq \sum_{n \in S} n x_{n}+\sum_{n \in S_{n}}\left(\sqrt{ } x_{n}\right)\left(\sqrt{ } x_{n}\right) \log \left(1 / x_{n}\right) .
$$

But $(\sqrt{ } t) \log (1 / t) \leq 2 e^{-1}$ for all $t>0$. Hence

$$
\begin{aligned}
\sum_{1}^{\infty} x_{n} \log \left(1 / x_{n}\right) & \leq \sum_{1}^{\infty} n x_{n}+2 e^{-1} \sum_{n \in S_{n}} \sqrt{ } x_{n} \\
& \leq \sum_{1}^{\infty} n x_{n}+2 e^{-1} \sum_{1}^{\infty} \exp \left(-\frac{1}{2} n\right) .
\end{aligned}
$$

LEMMA 2. If $\mu$ is a probability measure on $M$ and $\rho: M \rightarrow(0,1)$ is such that $\log \rho$ is $\mu$-integrable, then there exists a countable partition $\mathscr{P}$ of $M$ with entropy $H(\mathscr{P})<+\infty$ such that, if $\mathscr{P}(x)$ denotes the atom of $\mathscr{P}$ containing $x$, then

$$
\operatorname{diam} \mathscr{P}(x) \leq \rho(x)
$$

for a.e. $x \in M$. 
Proof. There exists $C>0$ and $r_{0}>0$ such that, for all $0<r \leq r_{0}$, there exists a partition $\mathscr{P}_{r}$ of $M$ whose atoms have diameter less than or equal to $r$ and such that the number of atoms in $\mathscr{P}_{r}$ (denoted by $\left.\left|\mathscr{P}_{r}\right|\right)$ satisfies

$$
\left|\mathscr{P}_{r}\right| \leq C(1 / r)^{\operatorname{dim} M} \text {. }
$$

Now define $U_{n}=\{x \mid \exp (-(n+1))<\rho(x) \leq \exp (-n)\}, \quad n \geq 0$. Since $\log \rho$ is integrable, we have

$$
\sum_{1}^{\infty} n \mu\left(U_{n}\right)<\infty
$$

By lemma 1,

$$
\sum_{1}^{\infty} \mu\left(U_{n}\right)\left(-\log \mu\left(U_{n}\right)\right)<+\infty
$$

Finally, define $\mathscr{P}$ as the partition given by the sets $Q \cap U_{n}$, with $n \geq 0$ and $Q \in \mathscr{P}_{r_{n}}$, $r_{n}=\exp (-(n+1))$, such that $\mu\left(Q \cap U_{n}\right) \neq 0$. Then

$$
H(\mathscr{P})=\sum_{n=0}^{\infty}\left(-\sum_{\substack{P \in \mathscr{P} \\ P \subset U_{n}}} \mu(P) \log \mu(P)\right) .
$$

Recalling the well known inequality

$$
-\sum_{i=1}^{m} x_{i} \log x_{i} \leq\left(\sum_{1}^{m} x_{i}\right)\left(\log m-\log \sum_{1}^{m} x_{i}\right)
$$

which holds for any set of real numbers $0<x_{i} \leq 1, i=1, \ldots, m$, we obtain

$$
\begin{aligned}
H(\mathscr{P}) & \leq \sum_{0}^{\infty} \mu\left(U_{n}\right)\left[\log \left|\mathscr{P}_{r_{n}}\right|-\log \mu\left(U_{n}\right)\right] \\
& \leq \sum_{0}^{\infty} \mu\left(U_{n}\right)\left[\log C+(\operatorname{dim} M)(n+1)-\log \mu\left(U_{n}\right)\right] .
\end{aligned}
$$

By (3), (4) and lemma 1, the last series converges and then $H(\mathscr{P})<+\infty$. Moreover, $x \in U_{n}$ implies that $\mathscr{P}(x)$ is contained in an atom of $\mathscr{P}_{r_{n}}$. Then $\operatorname{diam} \mathscr{P}(x)<r_{n}=$ $\exp (-(n+1))<\rho(x)$.

Proof of proposition. To prove the proposition take the partition $\mathscr{P}$ given by lemma 2. Denote by $\mathscr{P}_{n}(x)$ the atom of $\mathscr{P} \vee g^{-1}(\mathscr{P}) \vee \cdots \vee g^{-n}(\mathscr{P})$ which contains $x$. By the Shannon-McMillan-Breiman theorem,

$$
h_{\mu}(g) \geq h_{\mu}(\mathscr{P}, g)=\int_{M} \lim _{n \rightarrow+\infty} \frac{1}{n}\left[-\log \mu\left(\mathscr{P}_{n}(x)\right)\right] d \mu(x)
$$

To complete the proof we have to show that

$$
\lim _{n \rightarrow+\infty} \frac{1}{n}\left[-\log \mu\left(\mathscr{P}_{n}(x)\right)\right] \geq h_{\nu}(g, \rho, x) \quad \mu \text {-a.e. }
$$

Let $\mathscr{P}_{\infty}$ be the $\sigma$-algebra generated by the partitions $\mathscr{P}_{n}, n \geq 0$. Let $k: M \rightarrow \mathbb{R}$ be a $\nu$-integrable function, measurable with respect to the $\sigma$-algebra $\mathscr{P}_{\infty}$, such that

$$
\int_{A} k d \nu=\mu(A)
$$


for all $\boldsymbol{A} \in \mathscr{P}_{\infty}$. Such a function exists by the Radon-Nikodym theorem. It follows from (6) that

$$
\lim _{n \rightarrow+\infty} \frac{\mu\left(\mathscr{P}_{n}(x)\right)}{\nu\left(\mathscr{P}_{n}(x)\right)}=k(x) \quad \nu \text {-a.e. }
$$

Hence

$$
\frac{1}{n} \log \mu\left(\mathscr{P}_{n}(x)\right)=\frac{1}{n} \log \nu\left(\mathscr{P}_{n}(x)\right)+\frac{1}{n} \log \frac{\mu\left(\mathscr{P}_{n}(x)\right)}{\nu\left(\mathscr{P}_{n}(x)\right)}
$$

and, using (7), we obtain

$$
\lim _{n \rightarrow+\infty} \frac{1}{n}\left[-\log \mu\left(\mathscr{P}_{n}(x)\right)\right]=\lim _{n \rightarrow+\infty} \frac{1}{n}\left[-\log \nu\left(\mathscr{P}_{n}(x)\right)\right] \quad \mu \text {-a.e. }
$$

Moreover, it is easy to see that $\mathscr{P}_{n}(x) \subset S_{n}(g, \rho, x)$. Therefore, (5) follows from (9).

Before going into the proof of Pesin's formula we shall prove a technical lemma. The reader familiar with the Hadamard graph transform method for constructing invariant manifolds will recognize in this lemma one of the steps of that method. In the statement of the lemma we shall use the following definitions.

Definition. If $E$ is a normed space and $E=E_{1} \oplus E_{2}$ is a splitting, we define $\gamma\left(E_{1}, E_{2}\right)$ as the supremum of the norms of the projections $\pi_{i}: E \rightarrow E_{i}, i=1,2$, associated with the splitting. Moreover, we shall say that a subset $G \subset E$ is a $\left(E_{1}, E_{2}\right)$-graph if there exists an open $U \subset E_{2}$ and a $C^{1}$ map $\Psi: U \rightarrow E_{1}$ satisfying $G=\{x+\Psi(x) \mid x \in U\}$. The number $\sup \{\|\Psi(x)-\Psi(y)\| /\|x-y\| \mid x, y \in U\}$ will be called the dispersion of $G$.

LEMMA 3. Given $\lambda>\beta>1, \alpha>0$ and $c>0$, there exists $\delta>0$ with the following property. If $E$ is a finite-dimensional normed space and $E=E_{1} \oplus E_{2}$ a splitting with $\gamma\left(E_{1}, E_{2}\right) \leq \alpha$, and $F$ is a $C^{1}$ embedding of a ball $B_{r}(0) \subset E$ into another Banach space $E^{\prime}$ satisfying

(i) $D_{0} F$ is an isomorphism and $\gamma\left(\left(D_{0} F\right) E_{1},\left(D_{0} F\right) E_{2}\right) \leq \alpha$;

(ii) $\left\|\left(D_{0} F\right)-\left(D_{x} F\right)\right\| \leq \delta$ for all $x \in B_{r}(0)$;

(iii) $\left\|\left(D_{0} F\right) v\right\| \geq \lambda\|v\|$ for all $v \in E_{2}$;

(iv) $\left\|\left(D_{0} F\right) / E_{1}\right\| \leq \beta$;

then for every $\left(E_{1}, E_{2}\right)$-graph $G$ with dispersion $\leq c$ contained in the ball $B_{r}(0)$, its image $F(G)$ is a $\left(\left(D_{0} F\right) E_{1},\left(D_{0} F\right) E_{2}\right)$-graph with dispersion $\leq c$.

Proof. Identity $E$ with $E_{1} \times E_{2}$ and $E^{\prime}$ with $\left(D_{0} F\right) E_{1} \times\left(D_{0} F\right) E_{2}$. Write the map $F$ in the form

$$
F(x, y)=(L x+p(x, y), T y+q(x, y)),
$$

where $L=\left(D_{0} F\right) / E_{1}, T=\left(D_{0} F\right) / E_{2}$. It follows that the partial derivatives of $p$ and $q$ with respect to $x$ and $y$ have norm $\leq \delta \alpha$. Let $U \subset E_{2}$ be an open set and $\Psi: U \rightarrow E_{1}$ a map whose graph $\{(\Psi(v), v) \mid v \in U\}$ is $G$. Then,

$$
F(G)=\{(L \Psi(v)+p(\Psi(v), v), T v+q(\Psi(v), v)) \mid v \in U\} .
$$

To study this set define $\Phi: U \rightarrow\left(D_{0} F\right) E_{2}$ by

$$
\Phi(v)=T v+q(\Psi(v), v) .
$$


If $v, w \in U$,

$$
\|\Phi(v)-\Phi(w)\| \geq\|T(v-w)\|-\|q(\Psi(v), v)-q(\Psi(w), w)\| .
$$

Using the fact that the partial derivatives of $q$ are $\leq \delta \alpha$ and hypothesis (iii) we obtain

$$
\begin{aligned}
\|\Phi(v)-\Phi(w)\| & \geq \lambda\|v-w\|-\delta \alpha(\|\Psi(v)-\Psi(w)\|+\|v-w\|) \\
& \geq(\lambda-\delta \alpha(1+c))\|v-w\| .
\end{aligned}
$$

Hence, if $\delta$ is so small that $\lambda-\delta \alpha(1+c)>1, \Phi$ is a homeomorphism of $U$ onto $\Phi(U)$ whose inverse has Lipschitz constant $\leq(\lambda-\delta \alpha(1+c))^{-1}$. In particular, $\Phi(U)$ is open. Now define $\hat{\Psi}: \Phi(U) \rightarrow\left(D_{0} F\right) E_{1}$ by

$$
\hat{\Psi}(v)=\left(L \Psi \Phi^{-1}\right) v+p\left(\Psi\left(\Phi^{-1}(v)\right), \Phi^{-1}(v)\right) .
$$

Clearly, $F(G)=\{(\hat{\Psi}(x), x) \mid x \in \Phi(U)\}$. To calculate the dispersion of $F(G)$, write $\hat{\Psi}=\tilde{\Psi} \circ \Phi^{-1}$ where $\tilde{\Psi}(w)=L \Psi(w)+p(\Psi(w), w)$. Then

$$
\begin{aligned}
\|\tilde{\Psi}(x)-\tilde{\Psi}(y)\| & \leq \beta\|\Psi(x)-\Psi(y)\|+\delta \alpha(\|\Psi(x)-\Psi(y)\|+\|x-y\|) \\
& \leq(c \beta+\delta \alpha(1+c))\|x-y\| .
\end{aligned}
$$

Then the dispersion of $F(G)$ is less than or equal to

$$
c \frac{(\beta+\delta \alpha(1+c) / c)}{(\lambda-\delta \alpha(1+c))}=c \frac{\beta}{\lambda} \cdot \frac{(1+\delta \alpha(1+c) /(c \beta))}{(1-\delta \alpha(1+c) / \lambda)} .
$$

Since $\beta / \lambda<1$, taking $\delta$ small enough, the factor of $c$ is $<1$ and the lemma is proved.

Now we are ready to prove Pesin's formula. Let $f$ and $\mu$ satisfy the hypothesis of (2). Write

$$
E^{u}(x)=\oplus\left\{E_{j}(x) \mid \lambda_{j}(x)>0\right\}, \quad E^{0}(x)=\oplus\left\{E_{j}(x) \mid \lambda_{j}(x) \leq 0\right\} .
$$

Put $\Sigma_{j}=\left\{x \mid \operatorname{dim} E^{u}(x)=j\right\}$ and let $S=\left\{j \geq 0 \mid \mu\left(\Sigma_{j}\right)>0\right\}$. If $j \in S$, let $\mu_{i}$ be the measure on $M$ given by $\mu_{j}(A)=\mu\left(A \cap \Sigma_{i}\right) / \mu\left(\Sigma_{j}\right)$ if $A$ is a Borel subset of $M$. Then

$$
h_{\mu}(f)=\sum_{j \in S} \mu\left(\Sigma_{j}\right) h_{\mu_{j}}(f)
$$

Thus, all we have to show is that

$$
h_{\mu_{j}}(f) \geq \int_{M} \chi d \mu_{j}
$$

This inequality obviously holds for $j=0$. Suppose $j>0$. To simplify the notation put $\mu=\mu_{j}, \Sigma=\Sigma_{j}$. Fix any $\varepsilon>0$. By a standard combination of Egorov's theorem and Birkhoff's ergodic theorem, we can take a compact set $K \subset M$ with $\mu(K) \geq 1-\varepsilon$ such that the splitting $E^{0}(x) \oplus E^{u}(x)=T_{x} M$ is continuous when $x$ varies in $K$ and, for some $N>0$, there exist constants $\lambda>\beta>1$ such that, if $g=f^{N}$, the inequalities

$$
\begin{gathered}
\left\|\left(D_{x} g^{n}\right) v\right\| \geq \lambda^{n}\|v\| \\
\left\|\left(D_{x} g^{n}\right) / E^{0}(x)\right\| \leq \beta \\
\log \left|\operatorname{det}\left(D_{x} g^{n}\right) / E^{u}(x)\right| \geq N(\chi(x)-\varepsilon) n
\end{gathered}
$$

hold for all $x \in K, n \geq 0$ and $v \in E^{u}(x)$. In what follows, in order to avoid a cumbersome and conceptually unnecessary use of coordinate charts, we shall treat $M$ as if it were a Euclidean space. The reader will observe that all our arguments can be easily formalized by a completely straightforward use of local coordinates. 
LEMMA 4. For all $c>0$ there exists $\xi>0$ such that, if $x \in K$ and $g^{m}(x) \in K$ for some $m>0$, then if a set $G \subset M$ is contained in the ball $B_{\xi} m(x)$ and is a $\left(E^{0}(x), E^{u}(x)\right)$ graph with dispersion $\leq c$, then $g^{m}(G)$ is a $\left(\left(D_{x} g^{m}\right) E^{0}(x),\left(D_{x} g^{m}\right) E^{u}(x)\right)$-graph with dispersion $\leqslant c$.

Proof. Let $\alpha=\sup \left\{\gamma\left(E^{0}(x), E^{u}(x)\right) \mid x \in K\right\}$. Let $\delta>0$ be given by lemma 3 as a function of $\alpha, c$ and the constants $\beta, \lambda$ which satisfy (10) and (11). We claim that there exist $C>0$ and $0<t \leqslant 1$ such that

$$
\left\|\left(D_{x} g^{n}\right)-\left(D_{y} g^{n}\right)\right\| \leq C^{n}\|x-y\|^{t}
$$

for all $x, y \in M$. To prove this claim take $0<t \leq 1$ and $C_{0}>0$ satisfying $\left\|\left(D_{x} g\right)-\left(D_{y} g\right)\right\| \leq C_{0}\|x-y\|^{t}$ for all $x, y \in M$. Let $A$ be a Lipschitz constant for $g$. In particular, $A \geq\left\|\left(D_{x} g\right)\right\|$ for all $x \in M$. Choose $C$ satisfying for all $n \geq 0$

$$
C \geq A+C_{0}\left(\frac{A^{t+1}}{C}\right)^{n}
$$

then (13) holds for $n=1$. Suppose it holds for $n=1, \ldots, m$; then

$$
\begin{aligned}
\left\|\left(D_{x} g^{m+1}\right)-\left(D_{y} g^{m+1}\right)\right\| \leq & \left\|\left(D_{g^{m}(x)} g\right)-\left(D_{g^{m}(y)} g\right)\right\|\left\|\left(D_{y} g^{m}\right)\right\| \\
& +\left\|\left(D_{g^{m}(x)} g\right)\right\|\left\|\left(D_{x} g^{m}\right)-\left(D_{y} g^{m}\right)\right\| \\
\leq & A^{m} C_{0}\left\|g^{m}(x)-g^{m}(y)\right\|^{t}+A C^{m}\|x-y\|^{t} \\
\leq & A^{m} C_{0} A^{m t}\|x-y\|^{t}+A C^{m}\|x-y\|^{t} .
\end{aligned}
$$

By (14) the last expression is $\leq C^{m+1}\|x-y\|^{t}$, completing the proof of the claim. Now take $\xi>0$ such that $\left(C \xi^{t}\right)^{n}<\delta$ for all $n \geq 1$. Hence

$$
\begin{aligned}
\left\|\left(D_{y} g^{m}\right)-\left(D_{x} g^{m}\right)\right\| & \leq C^{m}\|x-y\|^{t} \\
& \leq C^{m} \xi^{m t}<\delta
\end{aligned}
$$

if $y \in B_{\xi} m(x)$. Therefore lemma 4 follows from lemma 3.

Fix the constant $c$ of the statement of lemma 4 so small that there exists $a>0$ with the following property. If $x \in K, y \in M$ and $d(x, y)<a$, then for every subspace $E \subset T_{y} M$ which is a $\left(E^{0}(x), E^{u}(x)\right)$-graph with dispersion $\leq c$ we have

$$
|\log | \operatorname{det}\left(D_{y} g\right) / E|-\log | \operatorname{det}\left(D_{x} g\right) / E^{u}(x)|| \leq \varepsilon .
$$

If $x \in K$, write

$$
D_{r}(x)=\left\{x+y_{1}+y_{2} \mid y_{1} \in E^{0}(x), y_{2} \in E^{u}(x),\left\|y_{1}\right\| \leq r,\left\|y_{2}\right\| \leq r\right\}
$$

and let $k_{2}>k_{1}>0, r_{1}>0$ be such that

$$
B_{k_{1 r}}(x) \subset D_{r}(x) \subset B_{k_{2} r}(x)
$$

for all $x \in K, 0<r \leq r_{1}$. Finally, if $x \in K$, define $N(x)$ as the minimum integer $\geq 1$ such that $g^{N(x)}(x) \in K$.

This function is well defined for a.e. $x \in K$ and is integrable because, if $W_{j}=$ $\{x \in K \mid N(x)=j\}$ it is easy to see that

$$
\bigcup_{n \geq 0} g^{n}(K)=\bigcup_{j=1}^{\infty} \bigcup_{i=0}^{j-1} g^{i}\left(W_{j}\right) \quad \bmod (0)
$$


and that the sets in the union on the right are disjoint. Hence,

$$
1>\mu\left(\bigcup_{n \geq 0} g^{n}(K)\right)=\sum_{j=1}^{\infty} \sum_{i=0}^{j-1} \mu\left(g^{i}\left(W_{j}\right)\right)=\sum_{j=1}^{\infty} j \mu\left(W_{j}\right)=\int_{M} N d \mu .
$$

Extend $N$ to $M$, putting $N(x)=0$ when $x \in K$, and define $\rho: M \rightarrow(0,1)$ as

$$
\rho(x)=\min \left(a,\left(k_{1} / k_{2}\right) \xi^{N(x)}\right) \text {. }
$$

The integrability of $N$ implies that of $\log \rho$. Let $\nu$ be the Lebesgue measure on $M$. We claim that there exists $K^{\prime} \subset K$ with $\mu\left(M-K^{\prime}\right) \leq 2 \sqrt{\varepsilon}$ such that

$$
h_{\nu}(g, \rho, x) \geq N(\chi(x)-\varepsilon-4 C \sqrt{ } \varepsilon)-\varepsilon
$$

for $\mu$-a.e. point $x \in K^{\prime}$, where $C=\sup \left\{\log \left|\operatorname{det}\left(D_{p} f\right) / E\right| \mid p \in M, E \subset T_{p} M\right\}$. By the proposition, this property will imply

$$
\begin{aligned}
h_{\mu}(g) & \geq \int_{M} h_{\nu}(g, \rho, x) d \mu(x) \geq \int_{K^{\prime}} h_{\nu}(x) \\
& \geq N \int_{K^{\prime}} \chi d \mu-N(\varepsilon+4 C \sqrt{ } \varepsilon)-\varepsilon \\
& \geq N \int_{M} \chi d \mu-N(\varepsilon+6 C \sqrt{ } \varepsilon)-\varepsilon
\end{aligned}
$$

Hence,

$$
h_{\mu}(f) \geq(1 / N) h_{\mu}(g) \geq \int_{M} \chi d \mu-(\varepsilon+6 C \sqrt{ } \varepsilon)-\varepsilon / N .
$$

Since $\varepsilon$ is arbitrary this completes the proof of (2).

It remains to prove the claim. Observe that Birkhoff's theorem implies

$$
\mu\left(\left\{x \mid \lim _{n \rightarrow+\infty}(1 / n) \#\left\{0 \leq j \leq n \mid g^{j}(x) \notin K\right\} \leq \sqrt{ } \varepsilon\right\}\right) \geq 1-\sqrt{ } \varepsilon .
$$

Then there exists a compact set $K_{1} \subset K$ with $\mu\left(K_{1}\right) \geq 1-2 \sqrt{\varepsilon}$ and $N_{0}>0$ such that, if $n>N_{0}$,

$$
\#\left\{0 \leq j \leq n \mid g^{i}(x) \notin K\right\} \leq 2 n \sqrt{ } \varepsilon
$$

for all $x \in K_{1}$. Define $K^{\prime}=K \cap K_{1}$. Clearly, $\mu\left(K-K^{\prime}\right) \leq \mu\left(M-K_{1}\right) \leq 2 \sqrt{ } \varepsilon$. Fix any $x \in K^{\prime}$. There exists $B>0$ satisfying

$$
\nu\left(S_{n}(g, \rho, x)\right)=B \int_{E^{0}(x)} \nu\left[\left(y+E^{u}(x)\right) \cap S_{n}(g, \rho, x)\right] d \nu(y)
$$

for all $n \geq 0$, where $\nu$ also denotes the Lebesgue measure in the subspaces $E^{0}(x)$, $y+E^{u}(x)$. Thus the claim is reduced to showing that

$$
\limsup _{n \rightarrow+\infty} \inf _{y \in E^{\circ}(x)} \frac{1}{n}\left[-\log \nu\left(\left(y+E^{u}(x)\right) \cap S_{n}(g, \rho, x)\right)\right] \geq N(\chi(x)-\varepsilon-4 C \sqrt{ } \varepsilon)-\varepsilon .
$$

If $y \in E^{0}(x)$, denote by $\Lambda_{n}(y)$ the set of points $w \in y+E^{u}(x)$ such that

$$
g^{i}(w) \in D_{\rho\left(g^{j}(x)\right) / k_{1}}\left(g^{j}(x)\right)
$$

for all $0 \leq j \leq n$. By $(16)$ we have

$$
\Lambda_{n}(y) \supset\left(y+E^{u}(x)\right) \cap S_{n}(g, \rho, x) .
$$


Therefore it is sufficient to prove that

$$
\limsup _{n \rightarrow+\infty} \inf _{y \in E^{\mathrm{o}}(x)} \frac{1}{n}\left[-\log \nu\left(\Lambda_{n}(y)\right)\right] \geq N(\chi(x)-\varepsilon-4 C \sqrt{\varepsilon})-\varepsilon .
$$

LEMMA 5. If $g^{n}(x) \in K$ and $y \in E^{0}(x)$ then, if $\Lambda_{n}(y)$ is not empty, $g^{n}\left(\Lambda_{n}(y)\right)$ is a $\left(E^{0}\left(g^{n}(x)\right), E^{u}\left(g^{n}(x)\right)\right)$-graph with dispersion $\leq c$.

Proof. The property is trivial for $n=0$. Suppose that it holds for some $m>0$. Then, if $g^{\bar{m}}(x) \in K$ and $g^{i}(x) \notin K$ for $m<j<\bar{m}$ and $\Lambda_{\bar{m}}(y) \neq \varnothing$, we have

$$
g^{\bar{m}}\left(\Lambda_{\bar{m}}(y)\right) \subset g^{\bar{m}-m}\left(g^{m}\left(\Lambda_{m}(y)\right)\right) \cap D_{\rho\left(g^{\bar{m}}(x)\right) / k_{1}}\left(g^{\bar{m}}(x)\right) .
$$

But $g^{m}\left(\Lambda_{m}(y)\right)$ is a $\left(E^{0}\left(g^{m}(x)\right), E^{u}\left(g^{m}(x)\right)\right)$-graph with dispersion $\leq c$, and

$$
\begin{aligned}
g^{m}\left(\Lambda_{m}(y)\right) & \subset D_{\rho\left(g^{m}(x)\right) / k_{1}}\left(g^{m}(x)\right) \\
& \subset B_{k_{2} \rho\left(g^{m}(x)\right) / k_{1}}\left(g^{m}(x)\right) \subset B_{\xi^{m-m}}\left(g^{m}(x)\right) .
\end{aligned}
$$

Then, using the fact that $N\left(g^{m}(x)\right)=\bar{m}-m$ and lemma 4, it follows that $g^{\bar{m}}\left(\Lambda_{m}(y)\right)=$ $g^{\bar{m}-m}\left(g^{m}\left(\Lambda_{m}(y)\right)\right)$ is a $\left(E^{0}\left(g^{\bar{m}}(x)\right), E^{u}\left(g^{\bar{m}}(x)\right)\right)$-graph with dispersion $\leq c$. Therefore, so is its open subset $g^{\bar{m}}\left(\Lambda_{\bar{m}}(y)\right)$.

To complete the proof of (18) take $D>0$ such that $D>\operatorname{vol}(G)$ (where vol( $\cdot$ ) denotes volume) for every $\left(E^{0}(w), E^{u}(w)\right)$-graph $G$ with dispersion $\leq c$ contained in $D_{\rho(w)}(w), w \in K$. Then, if $g^{n}(x) \in K$ and $y \in E^{0}(x)$, we have

$$
D>\operatorname{vol}\left(g^{n}\left(\Lambda_{n}(y)\right)\right)=\int_{\Lambda_{n}(y)}\left|\operatorname{det}\left(D_{z} g^{n}\right) / T_{z} \Lambda_{n}(y)\right| d \nu(z) .
$$

Let $S_{n}=\left\{0 \leq j<n \mid g^{j}(x) \in K\right\}$. If $n \geq N_{0}$ it follows from (15), (12), and (17) that $\log \left|\operatorname{det}\left(D_{z} g^{n}\right) / T_{z} \Lambda_{n}(y)\right|=\sum_{j=0}^{n-1} \log \left|\operatorname{det}\left(D_{g^{i}(z)} g\right) / T_{g^{j}(z)} g^{i}\left(\Lambda_{n}(y)\right)\right|$

$$
\begin{aligned}
& \geq \sum_{j \in S_{n}} \log \left|\operatorname{det}\left(D_{g^{i}(z)} g\right) / T_{g^{i}(z)} g^{j}\left(\Lambda_{n}(y)\right)\right|-N C\left(n-\# S_{n}\right) \\
& \geq \sum_{j \in S_{n}} \log \left|\operatorname{det}\left(D_{g^{i}(x)} g\right) / E^{u}\left(g^{i}(x)\right)\right|-\varepsilon n-N C\left(n-\# S_{n}\right) \\
& \geq \sum_{i=0}^{n-1} \log \mid \operatorname{det}\left(D_{g^{i}(x)} g\right) / E^{u}\left(g^{j}(x)\right)-\varepsilon n-2 N C\left(n-\not S_{n}\right) \\
& \geq n N(\chi(x)-\varepsilon)-\varepsilon n-2 N C\left(n-\# S_{n}\right) \\
& \geq n N(\chi(x)-\varepsilon)-\varepsilon n-4 N C n \sqrt{\varepsilon} .
\end{aligned}
$$

Then

$$
D>\nu\left(\Lambda_{n}(y)\right) \cdot \exp (n(N(\chi(x)-\varepsilon-4 C \sqrt{ } \varepsilon)))
$$

for all $y \in E^{0}(x), n \geq N_{0}$. This completes the proof of (18).

\section{REFERENCES}

[1] Y. Pesin. Characteristic Lyapunov exponents and smooth ergodic theory. Russian Math. Surveys 32 (1977), 55-114.

[2] D. Ruelle. An inequality for the entropy of differentiable maps. Bol. Soc. Bras. Mat. 9 (1978), 83-87. 